(C)2007 IEEE. Personal use of this material is permitted. However, permission to reprint/republish this material for advertising or promotional purposes or for creating new collective works for resale or redistribution to servers or lists, or to reuse any copyrighted component of this work in other works must be obtained from the IEEE 


\title{
Project Track and Trace Methodology in the Environment of Digital Ecosystem
}

\author{
Hai Dong ${ }^{1}$, Farookh Khadeer Hussain ${ }^{2}$ and Elizabeth Chang $^{3}$ \\ ${ }^{\text {l} H a i ~ D o n g, ~ D E B I I, ~ C u r t i n ~ U n i v e r s i t y ~ o f ~ T e c h n o l o g y, ~ A u s t r a l i a, ~ e-m a i l: ~ h a i . d o n g @ a p o s t g r a d . c u r t i n . e d u . a u ~}$ \\ ${ }^{2,3}$ Farookh Khadeer Hussain and Elizabeth Chang, School of Information Systems, Curtin University of Technology, Australia \\ e-mail: (Farookh.Hussain, Elizabeth.Chang)@.cbs.curtin.edu.au
}

\begin{abstract}
Digital Ecosystems (DE) is an open-source network environment for Small and Medium Enterprises (SMEs). By means of DE, project organisations are able to realize the distribution of organisational structure, to reduce internal cost and to achieve the biggest benefit. However, some issues exist in the process of project track and trace processes. In this paper, we analyze the daily project track and trace activities, including the organisational structure, the procedure of project planning and the scenarios of project distribution, by a case study in City of Melville Council - a typical project organisation, to discover the issues in these activities. To solve the issues, we extend the theory of CCCI Metrics to the field of project management. By means of the extended theory, a Project Track and Trace Card is designed to assist the senior management to timely administrate the project completion status in organisations, and then to promote the knowledge sharing in project organisations.
\end{abstract}

Index Terms-Digital Ecosystem, Knowledge Sharing, CCCI Metrics, Project Track and Trace.

\section{INTRODUCTION}

Digital Ecosystems (DE) is a neoteric terminology, which is an open-source network environment for business mainly including Small and Medium Enterprises (SMEs) to interact with others in an efficient way [2]. The appearance of DE is as a result of the natural existence of Business Ecosystem (BE) along with the evolution of business network and information technology. The goal of DE is to improve the efficiency of the communication between internal agents and to structuralize the existing BE. DE has the specification of "loosely-coupled, demand driven, domain clustered and agent-based" [3].

By means of DE, most organisations may improve cooperation between organisations or within organisations. To the latter, based on advanced communication technologies in DE, SMEs are able to realize the distribution of organisational structure, to reduce internal cost and to achieve the biggest benefit. Project organisation is such a typical instance. Many project organisations have already realized the multi-site structure [4]. In a normal project organisation, several projects are being under control by senior management. Thus, project track and trace activities between dispersed project groups and centralised senior management are essential, to keep the quality of projects and the competitive ability of enterprises. However, since there is not an available methodology to track and trace project quality from the perspective of senior management, some issues exist in the field of project management and harm organisational competitive ability

In this paper, by means of a case study, we will analyze the issues existing in current project track and trace activities in project organisations. Then the theory of CCCI Metrics will be introduced to solve the issues. Based on the theory, a Project Track and Trace Card will be constructed as the goal of the future ontology design.

\section{ISSUES IN PROJECT TRACK AND TRACE ACTIVITIES IN Project Organisations}

\section{A. Typical project track and trace activities in multi-site project organisations}

To deeply study project track and trace activities in project organizations, here we choose a typical project organization as the object of the research, which is City of Melville Council (www.melville.wa.gov.au).

In the typical project organization, the organizational structure is as Fig. 1. CEO is responsible for the whole organization which consists of several departments. Directors are responsible for departments which consist of several divisions. Managers are responsible for divisions which consist of several personnel.

As a project organization, to plan and implement projects is the primary task of the organization. In the City of Melville Council, the process of project planning can be drawn as:

Step 1: Normally projects are drafted by one or more than one directors of departments.

Step 2: Then the directors hand in the project drafts to the Council Board which consists of CEO and consultants.

Step 3: The Council Board will evaluate the project drafts.

Step 4: If the project drafts are agreed, the projects will be distributed by CEO to the directors who draft the projects.

About the project distribution, normally there are three scenarios which are:

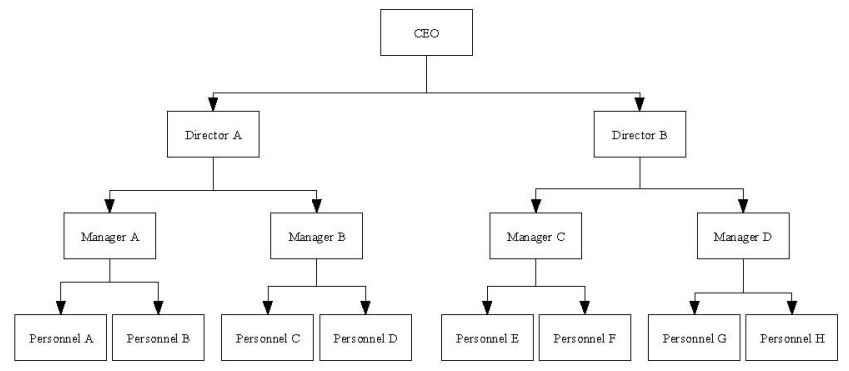


Fig. 1 Example of organizational structure

Scenario 1: CEO assigns one project to one department, then the director of the department is responsible for the project and divides it into several tasks for more than one managers. Each manager and related personnel act as the actual implementation of the tasks.

Scenario 2: CEO assigns several projects to one department, then the director of the department answers for the projects. The director assigns at least one project to each manager. Therefore, each manager is responsible for managing the implementation of corresponded projects.

Scenario3: CEO assigns one project to more than one department, and then the directors of the departments are responsible for the implementation of the project.

According to the above project planning steps and project distribution scenarios, we will examine the problematic situation of the project track and trace activities.

\section{B. Issues in the project track and trace activities}

The issues existing in project track and trace activities in City of Melville Council are as below:

- There is not existing methodologies to track and trace projects.

- CEO and Council Board do not know about the status of projects currently being processed in organizations.

- Directors cannot duly administrate the responsible projects owing to the lack of the necessary information about the projects.

- Managers cannot realize the real status of projects being implemented.

- If many people implement one project at the same time, they could not clearly distinguish their responsibilities.

- There is no criterion for assessing the completion of projects.

In conclusion, these issues could restrict the communication between senior management and each project group, which will affect the completion time and quality of projects. Thus, we proposed a project and track card, to solve the problems.

\section{Project Trace and Track Card}

According to the above issues, we design a Project Track and Trace Card based on the theory of CCCI Metrics, which is to track and trace the status of project completion from the perspective of management.

\section{A. Utilisation of CCCI Metrics in the field of project management}

CCCI Metrics [1] is utilized to measure the completion status of a project, which contains four elements as follows:

- Correlation of a project (CorrProject) - Degree of Comparison between the actual status of the project completion (ActualCompetionProject) and the mutually agreed status of the project completion (Mu- tually AgreedCompletionProject)

CorrProject $=($ ActualCompetionProject $) /$ (MutuallyAgreedCompletionProject)

- Correlation of a criterion (CorrCriterion) - A metric qualifies the extent of criterion completion in a project.

Extent: 0 - None/ Partially Completed

1 - Fully Competed (MaxCorrCriterion)

- Clarity of a criterion (ClearCriterion) - A metric qualifies the extent whether a criterion is mutually agreed between the evaluating person and the evaluated person or not.

Extent: 0 - This criterion is not mutually agreed between two sides.

1 - This criterion is mutually agreed between two sides.

- Importance of a criterion (ImpCriterion) - A Metric expresses the importance of a criterion.

Extent: $0-$ Not important

1 - Important

2 - Very important

Project Status $=$ (ActualCompetionProject) $/$ (Mutually AgreedCompletionProject $)=(($ CorrCriterion $1 *$ ClearCriterion $1^{*}$ ImpCriterion 1$)+\ldots \ldots+($ CorrCriterionN* ClearCriterionN* ImpCriterionN $))^{*} 6 / \quad($ MaxCorrCriterion1* ClearCriterion ${ }^{*}$ ImpCriterionl) $+\ldots \ldots+$ (MaxCorrCriterionN* ClearCriterionN* ImpCriterionN))

The scope of Project Status includes:

0 - Ignorance

1 - Completely unfinished

2 - Unfinished

3 - Minimally Finished

4 - Partially Finished

5 - Finished

6 - Completely finished

\section{B. Project Track and Trace Card}

The Project Track and Trace Card are composed of four parts (Fig. 2) which are:

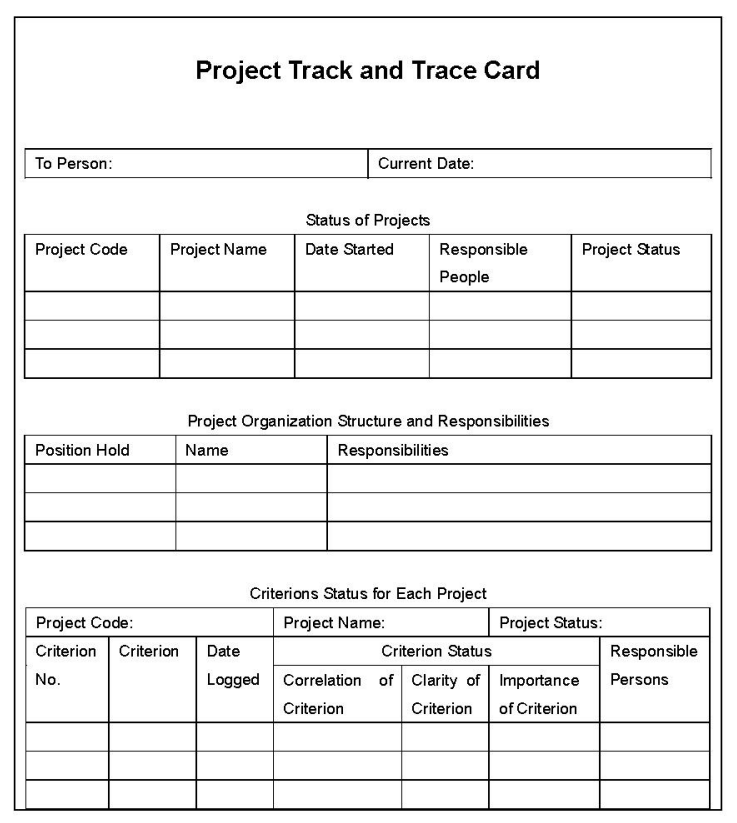


Fig. 2 Example of Project Track and Trace Card

The first part is to record the basic information of this card including the people who receive this card and the date when fills this card.

The second part is called "Status of Projects" which consists of five elements which are as follows, in the purpose of recording the basic information of projects.

- Project Code - the unique identifications of projects

- Project Name - the names of projects

- Date Started - the dates when projects begin

- Responsible People - the people who are responsible for a project

- Project Status - the measurement of projects completion which originates from CCCI Metrics

The third part is named as "Project Organization Structure and Responsibilities" which is to describe the structure of project organizations and the responsibilities of Employees. The elements are as follows:

- Position Hold - the positions of employees in a organizations

- Name - the names of employees

- Responsibilities - the projects that each employee is responsible to

The final part is "Criterion Status for each Project", which is to describe the basic information about criterions which are used to measure the completion of projects. The elements are as follows:

- Project Code, Project Name and Project Status - the basic information about the projects which criterions belongs to together

- Criterion No. - the unique identifications of criterions

- Criterion - the detailed information about criterions

- Date Logged - the date which a criterion is logged

- Criterion Status - the measurement of a criterion status which consists of three parts, namely "Correlation of Criterion", "Clarity of Criterion" and "Importance of Criterion" which are in correspondence with CCCI Metrics

- Responsible Persons - the persons who are responsible for a criterion

\section{Improved project distribution scenarios}

Based on the Project Track and Trace Card, the scenarios of project distribution in City of Melville are described as below:

Scenario 1: CEO assigns one project to one department, and then the director of the department divides the project into several tasks which are in correspondence with several criterions. These tasks which involve the criterions are being managed by several divisional managers.

Scenario 2: CEO assigns more than one projects to one Department, and then the director assign each divisional manager at least one project per person, which involves several tasks and corresponding criterions.

Scenario 3: CEO divides one project into several tasks which are in correspondence with several criterions, and then assigns these tasks to more than one directors of de- partment. Each director assigns more than one divisional manager at least one task per person.

\section{Project completion track and trace procedure}

With the appearance of Project Track and Trace Card, here we design the specific procedure for utilizing the card.

- If CEO wants to learn about the completion status of the current projects, CEO will ask the related directors to submit a Project Track and Trace Card.

- Then each director will fill in the card according to the actual status of each criterion contained in projects. Meanwhile, the director will evaluate the correlation, the clarity and the importance of the criterions by means of the quantitative method.

- After all directors submit the card about the projects under their supervision, $\mathrm{CEO}$ will utilize CCCI Metrics to measure the completion status of the projects

\section{Conclusion and Possible Future Works}

In the paper, by analyzing the organizational structure, the procedure of project planning and the module of project distribution scenarios in project organizations, we observe the problematic situation of project track and trace activities in the environment of DE. To solve the situation, we extend the theory of CCCI Metrics to the field of multi-site project management. Based on the theory, a Project Track and Trace Card is proposed to help senior management in organizations timely administrate the completion status of projects, in order to improve the competitive ability of project organizations.

The benefits of this project are concluded as below:

- It realizes the function of tracking and tracing projects completion status from the perspective of project management, which is beneficial to improving the competitive ability of project organizations.

- It simplifies the procedure of project track and trace activities and identifies own task and assessment criteria for each member in a project organization. Thus, the methodology may save the time and the cost of projects completion and help personnel self-evaluate.

The limitations of the project are concluded as below:

- The Project Track and Trace Card is not tested in practice, and thus we cannot validate its actual contribution to project track and trace activities in project organizations.

Based on the issues in the current project, in the future we will implement the card in real multi-site project organizations to validate its functions to project track and trace in the environment of DE. In addition, we will add more contents to the card according to different implementation situations.

\section{REFERENCES}

[1] E.Chang, T.S.Dillon and F.K.Hussain, Trust and Reputation for Service Oriented Environments-Technologies for Building Business Intelligence and Consumer Confidence, John Wiley \& Sons, Brisbane: 2005, pp.131-153. 
[2] E.Chang, M.Quaddus and R.Ramaseshan, The Vision of DEBI Institute: Digital Ecosystems and Business Intelligence, 2006.

[3] Digital Business Ecosystem: Introduction, Digital Business Ecosystem, 2006.
[4] J.J.J.Kasvi, M.Vartiainen and M.Hailikari, "Managing knowledge and knowledge competences in projects and project organisations," International Journal of Project Management, vol.21, no.8, 2003, pp $571-582$. 